\title{
Cost Design for Cross-layer Resource Allocation in Cognitive Radio Ad Hoc Networks
}

\author{
Wu Chunde $^{1} \quad$ Pan Zhiwen $^{1} \quad$ You Xiaohu $^{1} \quad$ Wu Ping $^{2} \quad$ Hu Honglin $^{3} \quad$ Zhang Yong $^{4}$ \\ 1 National Mobile Communications Research Laboratory, Southeast University, Nanjing, 210096, China \\ 2 Signals \& Systems, Dept. of Engineering Sciences, Uppsala University, Uppsala, Sweden \\ 3 Shanghai Research Center for Wireless Communications, Shanghai, China \\ 4 PCN\&CAD Certer, Beijing University of Posts and Telecommunications, Beijing, China
}

\begin{abstract}
In this paper, cost function for routing is studied. By introduction of improved cost function, an improved algorithm is proposed. Compared with the original algorithm, the improved algorithm is more stable and the feasible solution obtained is closer to the upper bound of optimal solution. The optimal solution lies between the upper bound and the feasible solution, so the feasible solution is even closer to the optimal solution. Therefore, algorithm with new cost function has better performance than the original algorithm.
\end{abstract}

Keywords- cognitive radio; ad hoc networks; cross-layer approach;link cost design

\section{INTRODUCTION}

Cognitive radio (CR) is an important technology for solving the spectrum underutilization problem. A cognitive radio has the capability to sense its environment and adapts its transmission parameter (e.g., carrier frequency, modulation, etc.)[1]. In a CR network there are two types of users: primary users and secondary users. The owner of a channel is referred to as primary users and all other users of the channel as secondary users. Secondary users identify the currently unused channels and accesses the channels opportunistically. By this way, the secondary users can share spectrum with the primary users. Different from the existing multi-channel multi-radio (MCMR) networks where the set of available channels at each node is identical (e.g., [2], [3], [4]), in CR networks the set of available channels is different from node to node. This adds complications to algorithm design for resource allocation in CR networks. Meanwhile, in ad hoc networks without a center controller, a distributed algorithm is needed.

In recent years, optimal resource allocation based on crosslayer approach has received enormous attention due to the increasing awareness that conventional network layering unnecessarily limits achievable network capacity (e.g., [5], [6]). Cross-layer approach integrates all resource from different layers to optimize resource allocation. The resource contains transmission power and code in physical layer, frequency schedule in MAC layer, routing in network layer. Especially in network layer, as an important aspect in cross-layer approach, link cost among nodes needs to be well designed for routing (e.g., [7]).
Hou et al. in [8] gave a definition of link cost named incremental link cost (ILC) and proposed a distributed resource allocation algorithm based on ILC. But according to their definition, when nodes choose route, they will only consider themselves in spite of the influence to their neighboring nodes. This results in instability of the distributed algorithm and even lack of feasible solution. In this paper, a improved link cost is proposed. Results show that improved resource allocation algorithm based on the new link cost function (LCF) provides better performance.

\section{ASSUMPTIONS AND NETWORK MODEL}

Consider a CR ad hoc network with $N$ secondary users. There are $M$ orthogonal channels in the network, denoted by the set $C$. Each secondary user individually detects available channels, and the set of available channels that can be used for communication is different from node to node. Let $C_{i}$ and $M_{i}$ denote the set and the number of available channels at node $i$, respectively. For each available frequency band at a node, the bandwidth is assumed to be $w$.

For data transmission from node $i$ to node $j$, a widely used model for power propagation gain $g_{i j}$ is

$$
g_{i j}=\frac{1}{d_{i j}{ }^{\alpha}} \text {. }
$$

Where $d_{i j}$ is the physical distance between nodes $i$ and $j$ and $\alpha$ is the path loss index.

Denote $p_{i j}^{m}$ the transmission power from node $i$ to node $j$ in frequency band $m$. Then the received power $p_{r}$ on node $j$ is

$$
p_{r}=p_{i j}^{m} \cdot g_{i j}=\frac{p_{i j}^{m}}{d_{i j}{ }^{\alpha}} .
$$

Assume a data transmission from node $i$ to node $j$ is successful only if the received power at node $j$ exceeds a power threshold, say $P_{T}$. Then we have

$$
p_{i j}^{m} \cdot g_{i j} \geq P_{T}
$$

This work is supported by International Science and Technology Cooperation Program under grant 2008DFA12090 and National Communication Research Laboratory Program (2008A02) 
Therefore, the minimum required transmission power $P_{i j}^{T}$ on node $i$ is

$$
P_{i j}^{T}=\frac{P_{T}}{g_{i j}}=P_{T} \cdot d_{i j}^{\alpha} .
$$

Similarly, it is assumed that an interference power is negligible only if it cannot exceed a threshold defined as $P_{I}$ at a receiver. Then we can infer that the interference radius of node $i$ is $\left(\frac{p_{i j}^{m}}{P_{I}}\right)^{1 / \alpha}$.

\section{DEFINITION OF COST FUNCTION}

In this section, we define the link cost function (LCF) and band cost function (BCF). According to the definition of link cost in [8], we firstly need to give the definition of band cost function (BCF). Then using BCF, we can define LCF.

\section{A. Band Cost Function (BCF)}

When a node uses a certain band to transmit, it must occupy some spectrum and space. The spectrum and space is the cost the node needs to pay for communication. A metric is proposed in [9] to represent the cost as space-bandwidth-utilization. While in [10] it is named bandwidth-footprint-product (BFP). Here we also call it as BFP. When a node uses some band to transmit, the BFP equals the production of bandwidth and interference area corresponding to its transmission power. So BFP characterizes the spectrum and space occupancy for a CR network. The smaller the total BFP of a network, the higher the utilization of spectrum and space.

For example, if node $i$ transmits data to node $j$ with transmission power $p_{i j}^{m}$ using band $m \in M_{i}$, the BFP is $w \bullet \pi \bullet\left(\frac{p_{i j}^{m}}{P_{I}}\right)^{2 / \alpha}$. Since each band has the same bandwidth, BFP reduces to $\pi \cdot\left(\frac{p_{i j}^{m}}{P_{I}}\right)^{2 / \alpha}$.

Based on BFP, we can define band cost function (BCF) as the ratio of the change of BFP and capacity in a certain band. On the assumption that node $i$ transmits data to node $j$ with transmission power $p_{i j}^{m}$ using band $m \in M_{i}$ in AWGN channel. The ambient Gaussian noise density is $\eta$. When band $m$ stays in different cases, the computation of BCF has following three ways:

First case, band $m$ is already used but $p_{i j}^{m}<\left(p_{i j}^{m}\right)_{U}$, where $\left(p_{i j}^{m}\right)_{U}$ is the maximum allowed transmission power of node $i$. BCF in band $m$ can be computed as

$$
B C F(i, j, m)=\frac{\pi \cdot\left[\left(p_{i j}^{m}\right)_{U} / P_{I}\right]^{2 / \alpha}-\pi \cdot\left(p_{i j}^{m} / P_{I}\right)^{2 / \alpha}}{w\left[\log _{2}\left[1+\frac{g_{i j}}{\eta w}\left(p_{i j}^{m}\right)_{U}\right]-\log _{2}\left(1+\frac{g_{i j}}{\eta w} p_{i j}^{m}\right)\right]} \cdot
$$

Second case, band $m$ is not yet used. BCF can be computed as

$$
\operatorname{BCF}(i, j, m)=\frac{\pi \cdot\left(P_{i j}^{T} / P_{I}\right)^{2 / \alpha}}{w \log _{2}\left(1+\frac{g_{i j}}{\eta w} P_{i j}^{T}\right)} .
$$

Third case, band $m$ is already fully utilized, i.e. $p_{i j}^{m}=\left(p_{i j}^{m}\right)_{U}$. BCF can be defined as $\infty$.

Foregoing definition of BCF is the same as incremental band cost (IBC) in [8]. Observing the definition in the second case, we can see that BCF is irrelative with $m$. That means for any band which is not yet used between node $i$ and node $j$, the BCF is the same. But actually, the influence of using different band to neighboring nodes is different. The neighboring nodes of a node refer to the nodes that fall in the transmission range of that node.

Take an example in Figure 1 where every node has a set of available bands. If node $\mathrm{A}$ transmits to node $\mathrm{B}$ using band 2 and node $\mathrm{C}$ falls in the interference range of node $\mathrm{A}$, then node $\mathrm{C}$ can not use band 2 . As a result, node $\mathrm{C}$ and node $\mathrm{D}$ can not communicate with each other. But if node A transmits to node B using band 1 , then node $\mathrm{C}$ and node $\mathrm{D}$ can communicate with each other using band 2 . So in this example, using band 1 or using band 2 for node $\mathrm{A}$ and node $\mathrm{B}$ will have different effect to neighboring nodes $\mathrm{C}$ and $\mathrm{D}$. Then naturally, BCF of band 1 should be different from that of band 2 .
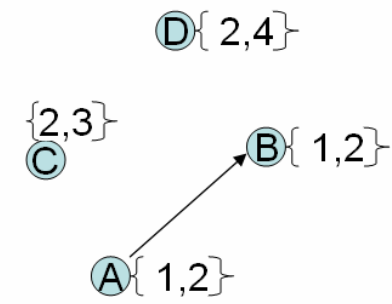

Figure 1. An Example of Band Difference

From the upper example, we can come to a conclusion that the definition of BCF in the second case must consider the influence to neighboring nodes. We define the influence of a certain band as the number of neighboring nodes which own that band. So the influence can be expressed as

$$
\operatorname{num}(i, m)=\sum_{j \in \mathrm{T}_{i}} \operatorname{link}(i, j, m) \text {. }
$$

Where $T_{i}$ is the set of neighboring nodes of node $i$, $\operatorname{link}(i, j, m)$ is $0-1$ binary variable. If node $i$ can use band $m$ to communicate with node $j$, then $\operatorname{link}(i, j, m)=1$.Otherwise, $\operatorname{link}(i, j, m)=0$.

Meanwhile, according to the node order proposed in [11], we realize that the more bands a node can use, the more flexible the node is in the aspect of band choosing, i.e. the less constraints on the node. That means it has more possibilities to add rate of sessions across the node. Hence, the definition of BCF in the second case should take into account the number of available bands in a node, i.e. $M_{i}$.

Jointly considering the upper two suggestions, we redefine the BCF in the second case as 


$$
B C F(i, j, m)=\frac{\pi \cdot\left(P_{i j}^{T} / P_{I}\right)^{2 / \alpha}}{w \log _{2}\left(1+\frac{g_{i j}}{\eta w} P_{i j}^{T}\right)} \cdot \frac{\operatorname{num}(i, m)+n u m(j, m)}{M_{i}+M_{j}} .
$$

\section{B. Link Cost Function (LCF)}

Based on BCF, the definition of link cost function (LCF) can be given. When there exists first case bands between node $i$ and node $j$ which are denoted by the set $A$, LCF can be defined as

$$
\operatorname{LCF}(i, j)=\min _{m \in A}\{B C F(i, j, m)\} .
$$

Otherwise, we examine if there exist second case bands, i.e. a new band. If exist those bands which are denoted by the set $B$, there are two definitions of BCF, i.e. (6) and (8). (6) just needs information of the transmission node, but (8) needs not only information of the transmission node but also information of the reception node. No matter which definition BCF is, LCF has definition as

$$
L C F(i, j)=\min _{m \in B}\{B C F(i, j, m)\} .
$$

If no band of the upper two cases exists, the LCF between node $i$ and node $j$ can be defined as

$$
\operatorname{LCF}(i, j)=\infty \text {. }
$$

\section{PERFormance EVAlUATION}

In this section, we present simulation results to demonstrate the performance of the new definitions of BCF and LCF by the distributed optimization algorithm proposed in [8].The objective of the algorithm is maximizing the minimum scaling factor of data rates for a set of user communication sessions. The detailed algorithm can be referred to [8].

\section{A. Simulation Setting}

Consider a 20-node ad hoc network with each node randomly located in a $60 \times 60$ area. The units for distance, rate, and power density are all normalized with appropriate dimensions. Assume there are $M=10$ frequency bands in the network and each band has a bandwidth of $w=50$. Each node may only have a subset of these frequency bands. In the simulation, this is done by randomly selecting a subset of bands for each node from the pool of 10 bands.

We assume that, under maximum transmission power, the transmission range on each node is 20 and the interference range is 30 . The pass loss index $\alpha$ is assumed to be 4 . The transmission threshold $P_{T}$ is assumed to be $\eta w$. Thus, we have the maximum transmission power $P_{\max }=20^{\alpha} \cdot P_{T}$ and the interference threshold $P_{I}=P_{T} /(1.5)^{\alpha}$.We assume there are 4 communication sessions, where the source and destination are randomly selected and the minimum rate requirement of each session is 10 .

\section{B. Simulation Results}

The metric of performance is the same as that used in [8], i.e. the ratio of scaling factor $K$ obtained via the distributed algorithm over that obtained by the upper bound. We call the original algorithm using ILC as link cost proposed in [8] ILCbased Algorithm (ILCA), the improved algorithm using our link cost LCF-based Algorithm (LCFA), i.e. ILCA uses the definition (6) and LCFA uses the definition (8). Besides, when choosing a new band, the criterions of the two algorithms are different. ILCA chooses the band with maximal $\left(p_{i j}^{m}\right)_{U}$, while LCFA chooses the band with minimal BCF. Figure 2 is the results obtained by ILCA and LCFA for 10 simulation runs respectively.

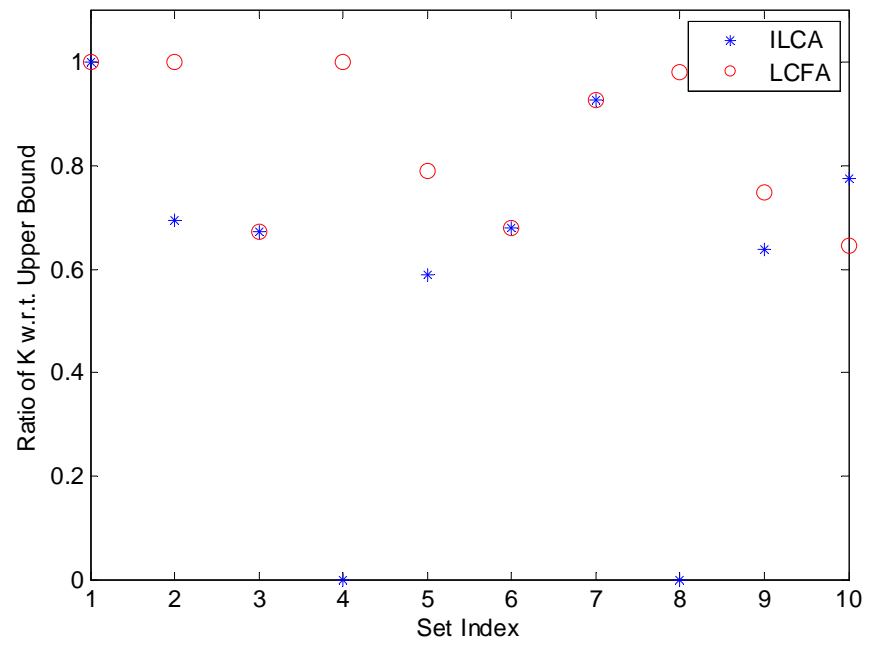

Figure 2. Results of ILCA and LCFA for 10 simulation runs

Figure 2 shows, as a whole, the solution of LCFA is closer to the upper bound than that of ILCA, i.e. closer to the optimal solution of centralized algorithm. That is because when choosing a new band, LCFA chooses the band with minimal BCF. That means the band have maximal potential capacity with minimal interference. So the session of LCFA can achieve higher rate, i.e. higher ratio.

Meanwhile, From Figure 2, we can see that some ratios of ILCA are zero. However, with the same parameters, the results of LCFA are all nonzero. For seeing that more clearly, now we count the percent of simulation runs whose ratio is zero for 100 simulation runs. Figure 3 shows the percent varying with the number of frequency bands $M$ from 3 to 10 for both algorithms. 


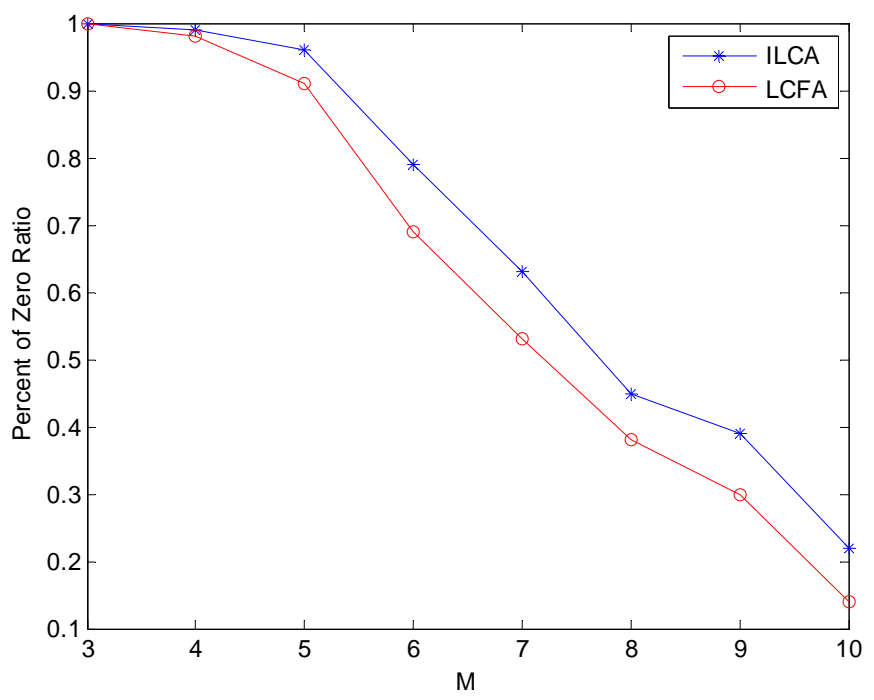

Figure 3. The percent of simulation runs whose ratio is zero varying with $M$

In Figure 3, the two percents decrease when $M$ increases. But the percent of LCFA is always smaller than the one of ILCA. That means LCFA is better in finding routes for sessions. We can explain that ILCA uses the definition (6) which does not consider the influence to neighboring nodes. As a result, when choosing route, a session choose a busy route which is the only route of another session. Then that session has no route to choose. So the ratio of the session is zero. Differently, LCFA uses the definition (8) which considers the potential interference to neighboring nodes. So the session will avoid choosing the route which is possible to collide with other sessions.

\section{CONCLUSION}

In this paper, we considered the cross-layer approach for resource allocation in cognitive radio ad hoc networks. Cost design for routing is necessary when network layer is taken into account. An improved cost function is presented. Two distributed algorithms, i.e. with original cost function and with improved cost function respectively, are used in the simulation. Numerical results show the new algorithm with improved cost function is more stable and the solution is closer to the optimal solution of centralized algorithm.

\section{ACKNOWLEDGMENT}

This work is supported by International Science and Technology Cooperation Program under grant 2008DFA12090 and National Communication Research Laboratory Program (2008A02).

\section{REFERENCES}

[1] Notice of Proposed Rule Making and Order, FCC. Et Docket no. 03-322, December 2003.

[2] M. Kodialam and T. Nandagopal, "Characterizing the capacity region in multi-radio multi-channel wireless mesh networks," Proc of the 11th annual international conference on Mobile computing and networking, Cologne, Germany, 2005, pp. 73-87.

[3] R. Draves, J. Padhye, and B. Zill, "Routing in multi-radio, multihop wireless mesh networks,"Proc of Proceedings of the 10th annual international conference on Mobile computing and networking, Philadelphia, PA, USA, 2004,pp.114-128.

[4] P. Kyasanur and N.H. Vaidya, "Capacity of multi-channel wireless networks: impact of number of channels and interfaces,"Proc of the 11th annual international conference on Mobile computing and networking, Cologne, Germany, 2005,pp.43-57.

[5] Miao Ma and Danny H. K. Tsang. ”Joint Spectrum Sharing and Fair Routing in Cognitive Radio Networks,"Proc of the 5th IEEE on Consumer Communications and Networking Conference -CCNC, 2008:978-982.

[6] Alex Fridman, Steven Weber, Kapil R. Dandekar, Moshe Kam,”Crosslayer multicommodity capacity expansion on ad hoc wireless networks of cognitive radios," Proc of the 42nd Annual Conference on Information Sciences and Systems,2008,pp. 676-680.

[7] Miao Pan, Rongsheng Huang, Yuguang Fang,"Cost design for opportunistic multi-hop routing in Cognitive Radio networks," Proc of IEEE Military Communications Conference, 2008,pp.1-7.

[8] Y. Shi and Y.T. Hou, "A distributed optimization algorithm for multihop cognitive radio networks,” Proc of the 27th IEEE International Conference on Computer Communications,2008,pp.1967-1974. .

[9] X. Liu and W. Wang, “On the characteristics of spectrum-agile communication networks,"Proc of the First IEEE International Symposium on New Frontiers in Dynamic Spectrum Access NetworksDySPAN,2005,pp.214-223.

[10] Y. Shi and Y. T. Hou, "Optimal power control for multi-hop software defined radio networks,"Proc of the 26th IEEE International Conference on Computer Communications,2007,pp.1694-1702.

[11] M. Thoppian, S. Venkatesan, R. Prakash, R. Chandrasekaran, "MAClayer scheduling in cognitive radio based multi-hop wireless networks,"Proc of the International Symposium on a World of Wireless, Mobile and Multimedia Networks, 2006,pp.193-202. 